\title{
sciendo
}

\section{FUNCTIONING OF THE INTESTINAL ECOSYSTEM: FROM NEW TECHNOLOGIES IN MICROBIAL RESEARCH TO PRACTICAL POULTRY FEEDING - A REVIEW*}

\author{
Zenon Zduńczyk \\ Institute of Animal Reproduction and Food Research, Polish Academy of Sciences, Tuwima 10, \\ 10-748 Olsztyn, Poland
}

\begin{abstract}
Unlike classical microbiology which focuses on bacteria capable of growing in vitro, metagenomics is a study of genetic information originating from microflora which aims to characterise the microbiome, namely the common genome of bacteria, archaea, fungi, protozoa and viruses living in the host. Metagenomics relies on next-generation sequencing (NGS), a large-scale sequencing technique which allows millions of sequential reactions to be carried out in parallel to decode entire communities of microorganisms. Metagenomic analyses support taxonomic analyses (involving gene fragments encoding ribosomal RNAs $5 \mathrm{~S}$ and $16 \mathrm{~S}$ in bacteria) or functional analyses for identifying genes encoding proteins that participate in the regulation of metabolic pathways in the body. New metagenomics technologies expand our knowledge of the phylogenetic structure of microflora in the gastrointestinal tract of poultry, and they support the identification of previously unknown groups of microbiota, mainly those occurring in small numbers. Next-generation sequencing also provides indirect information about the quantitative structure of the genes of gut microorganisms, but microbial activity and changes in the proportions of microbial metabolites that affect the host's intestinal integrity and metabolism remain insufficiently investigated. Therefore, research studies are undertaken to investigate the proportions of the key microbial metabolites in the intestinal contents of poultry relative to changes in the population size of the most important bacterial groups, including those determined by cheaper techniques.
\end{abstract}

Key words: intestinal microbiota, metagenomics, next-generation sequencing, intestinal ecosystem, poultry nutrition

"We are in the midst of what may, in retrospect, come to be referred to as the golden age of microbial ecology. The microorganisms and their genes associated with higher organisms (the microbiome) that were once viewed primarily as sources of human pathogens are now recognised as complex communities with an important influence on the health and disease status of the host". This is how certain authors (Oakley et al., 2014) assess the effects of the five-year research programme into the human microbiome, funded by the US National Institute of Health in 2007-2012. The term "microbiome" was suggested by the Nobel Prize laureate Joshua Lederberg to describe the collective genome of all commensal, synbiotic and pathogenic microorganisms found in the human body, in the gastrointestinal tract, on the skin, and in urinary and respiratory systems (Hooper and Gordon, 2001). At present, this term denotes the collective genome of microorganisms, i.e. bacteria, archaea, fungi,

*Study funded by Institute of Animal Reproduction and Food Research. 
protozoa and viruses living in the host's body, which undergo changes during the host's life (e.g. in response to the diet, environment, stress, medical interventions or medical conditions). The microbiome makes an important contribution to energy homeostasis, metabolism, health, immunological activity and neurodevelopment of the host's body (Turnbaugh et al., 2007; Cho and Blaser, 2012).

As at the end of 2018, over 20,000 scientific reports with the keyword "microbiome" had been published in the Web of Science. These reports have been published in the last 15 years, with 106, 2724 and 17357 works in subsequent five-year periods. This rapid increase in the number of scientific papers testifies to the growing interest in the microbiome. More than 100 papers focused on the microbiome of the gastrointestinal tract in poultry, whereas the remaining articles addressed diverse issues. The aim of this review article was to describe the potential scientific developments stemming from advances in metagenomics relative to classical microbiology, and to highlight the contribution of innovative research technologies to the acquisition of new knowledge about the composition and physiological impact of gastrointestinal microbiota on the body.

\section{Metagenomics: a new chapter in microbiology}

Metagenomics studies genetic material from environmental or host-associated microbiota to describe microbial diversity and function (Choi et al., 2015). Metagenomics emerged as a separate field of research in the last decades of the 20th century when more effective methods of cloning and identifying the DNA of microbial samples from the environment, initially soil, had been developed (Handelsman et al., 1998). Technological progress has led to the development of next-generation sequencing (NGS), a massive parallel sequencing approach for decoding entire microbial communities (Thomas et al., 2012). Microbiology has been revolutionised by technological advancement and high-performance metagenomics technologies involving direct cloning, sequencing and functional analyses of genetic material, as well as by the progress in bioinformatics, including the development of computational methods for analysing the structure, functions and evolution of genes, genomes and proteins (Borda-Molina et al., 2018; Barko et al., 2018).

Metagenomics researchers seek answers to the following questions: "Who is there?", which corresponds to phylogenetic profiling (taxonomic analyses), and "What are they doing?", which relates to genetic analyses (functional analyses) (Chistoserdova, 2009). The sequences of the genes encoding 5S and 16S ribosomal RNA, the molecular determinants of microbial phylogenesis, play a crucial role in taxonomic analyses of bacteria and archaea (Schloss and Handelsman, 2003). Different fragments of the ribosomal gene are amplified in other microorganisms, including the 18S rRNA gene in eukaryotic species and the nuclear ribosomal internal transcribed spacer regions in fungi (Meyer et al., 2010). In comparison with traditional microbiological techniques, microbial genera and species can be identified much more accurately based on detailed analyses of nucleotide subunits and comparisons with 16S rRNA gene sequences deposited in public databases (Deusch et al., 2015). Metagenomic functional analyses are an essential element of phylogenetic research and support the search for functional interactions between microbial species colonising specific environments (Campanaro et al., 2016). Functional analyses of the 
metagenome enable the determination of protein-encoding genes which participate in the control of the host's metabolic pathways. Gene functions are identified with the involvement of traditional methods, including analyses of gene homology (predicting gene functions by comparison with the structure of the recognised genes), gene inactivation and overexpression, as well as micromatrices and analyses of DNA microcracks with the use of bioinformatic tools (Sitnicka et al., 2010). Despite continuous progress in research, the acquisition of complete single genomes from metagenomic sequences still poses a challenge (Nielsen et al., 2014). Functional groups and bacterial taxa can be identified with the use of basic tools databases, such as the Kyoto Encyclopaedia of Genes and Genomes (KEGG). One of the limitations of this procedure stems from the fact that the diversity of genomes in nature is far greater than in limited sequence databases, which is why not all predicted genes will exhibit homology with known sequences (Sharpton, 2014).

Two distinct metagenomics approaches are commonly used: the first is referred to as marker-gene metagenomics or targeted metagenomics, and the second is known as shotgun metagenomics. In the past, the method of sequencing entire microbial DNA was more common. Shotgun metagenomics ("random firing pattern") offers a simpler solution by sequencing a large number of random sections of fragmented genomic DNA which are subsequently combined into continuous threads based on overlaps (overlapping regions) (Sharpton, 2014). This approach requires innovative computer-based computational methods to combine hundreds of thousands of short, randomly-obtained DNA sequences into longer, continuous fragments. Metagenomic analyses of the DNA pool of microorganisms colonising the bovine gut in a given environment also support the identification of genes encoding proteins that participate in the regulation of host metabolic pathways. Shotgun DNA sampling supports more efficient determination of functional metabolic profiles in bacterial communities than targeted metagenomics (Deusch et al., 2015).

The most important differences between the methods and results of classical microbiology and metagenomics are presented in Table 1. In classical microbiology, the microflora is regarded as a population of live microorganisms (colony forming units), whereas the term microbiome refers to the collective content of genomic microflora, where species-equivalent operational taxonomic units (OTUs) can be distinguished (Cole et al., 2014). An OTU is an organizational proxy for a species created by statistical clustering of units with high sequence similarity, typically higher than $97 \%$. The number of OTUs is disproportionately larger because OTUs account for more complex microflora (fungi, viruses as well as microbial expression products), including anaerobic microflora.

Several types of NGS techniques can be identified, depending on the applied analytical system (e.g. Illumina, ThermoFisher, and Bioscience) with different combinations of read length and read numbers that determine the system's throughput and the number of bases per run. Therefore, various analytical platforms can produce different results (Stanley et al., 2013; Ranjitkar et al., 2016). This problem has been discussed in other review articles (Liu et al., 2012; Scholz et al., 2012; Mohinudeen et al., 2017; Vincent et al., 2017). According to Nielsen et al. (2014), bacterial genomes from different isolates of the same species usually show considerable genetic 
heterogeneity. The results of microbiome analyses are also influenced by the method of sampling intestinal contents where samples can be collected individually from chickens or pooled from several birds. Errors are also encountered during sample preparation and storage, DNA extraction and read sequencing (Kunin et al., 2010; Cruaud et al., 2014; Hang et al., 2014). These issues are not discussed in this article.

Table 1. A brief comparison of conventional microbiological methods and metagenomics techniques (Salanitro et al., 1974; Tremaroli and Bäckhed, 2012; Wang et al., 2015; Barko et al., 2018; Borda-Molina et al., 2018)

\begin{tabular}{l} 
Classical microbiology \\
\hline - Enumeration of selected bacterial groups \\
cultured on selective media \\
- Determination of bacterial properties: \\
- morphology and structure of the cell wall \\
(Gram+/-), \\
- responses to oxygen, antibiotic resistance, \\
antibacterial activity immunomodulatory \\
effects, etc., \\
- fermentation activity: substrates and main \\
metabolites
\end{tabular}

\section{Methods}

- Analyses of nucleic acids - selection of genetic material for the entire microbial population

- Determination of the structure and physiological functions of microbiota:

- taxonomic analyses of bacterial populations (sequences encoding $16 \mathrm{~S}$ rRNA)

- functional analyses to determine the presence of genes encoding specific enzymatic/metabolic activity

Results

- Visible but only culturable bacterial colonies (approximately $1-10 \%$ of bacteria in the gatrointestinal tract)

- Bacterial counts expressed in CFU/g or CFU/ $\mathrm{ml}$

- All bacteria, fungi, archaea, viruses and their gene products present in the environment

- Composition of microbiota expressed by the number of species-equivalent operational taxonomic units (OTUs)

\section{Applications}

- Isolation of target bacterial colonies

- Determination of bacterial diversity

- Clinical diagnosis

- Determination of microbial dysbiosis

\section{Metagenomic analyses of poultry gut microbiota}

Earlier studies show that bacterial counts in the ileum and caeca of day-old chicks can reach $10^{8}$ and $10^{10} \mathrm{CFU}$ per $\mathrm{g}$ of intestinal contents, respectively (Apajalahti et al., 2004). Fifty species of bacteria were identified in day-old chicks, and the number of bacterial species in caecal digesta increased to 200 in 42-day-old chicks (Oakley et al., 2014). The rate of microbial colonisation in the gastrointestinal tract is determined by hygiene standards, diet composition and feed intake. In three-weekold chicks, the proportion of lactic acid bacteria in ileal microbiota exceeded $70 \%$, whereas the proportion of other bacterial groups, including Clostridium spp., Streptococcus spp. and Enterobacteriaceae spp., was considerably smaller. In the final rearing stage, significant variations were noted in the composition of the microbiota colonising the small intestine and the caeca. In quantitative terms, Lactobacillus bacteria were predominant in the ileum, whereas the proportion of Clostridium did not exceed $10 \%$. The reverse proportions of Lactobacillus and Clostridium were observed in the caeca (7.75 vs 39.3\%) (Lu et al., 2003). Both intestinal segments generally differ in microbial counts, with $10^{8}-10^{9} \mathrm{CFU}$ in the ileum, and $10^{10}-10^{11}$ CFU in the caeca (Yeoman et al., 2012). 


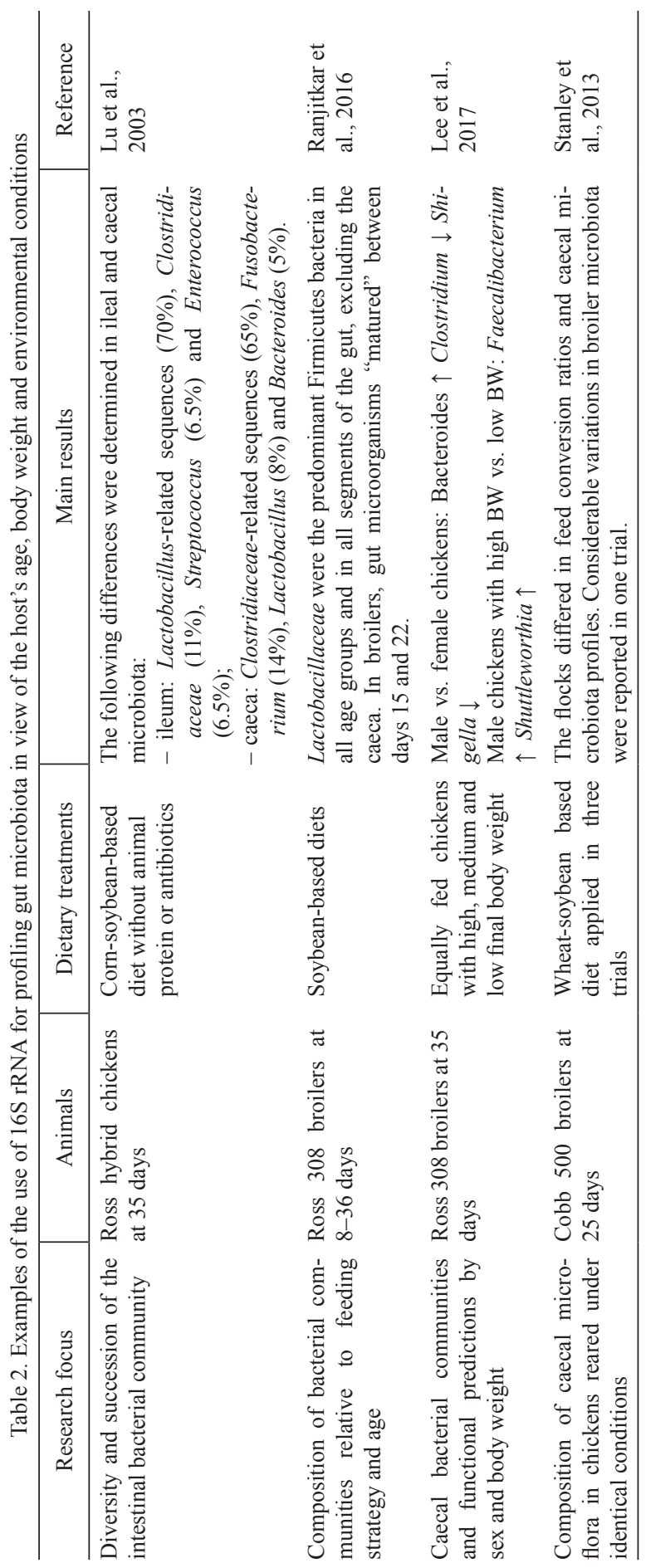




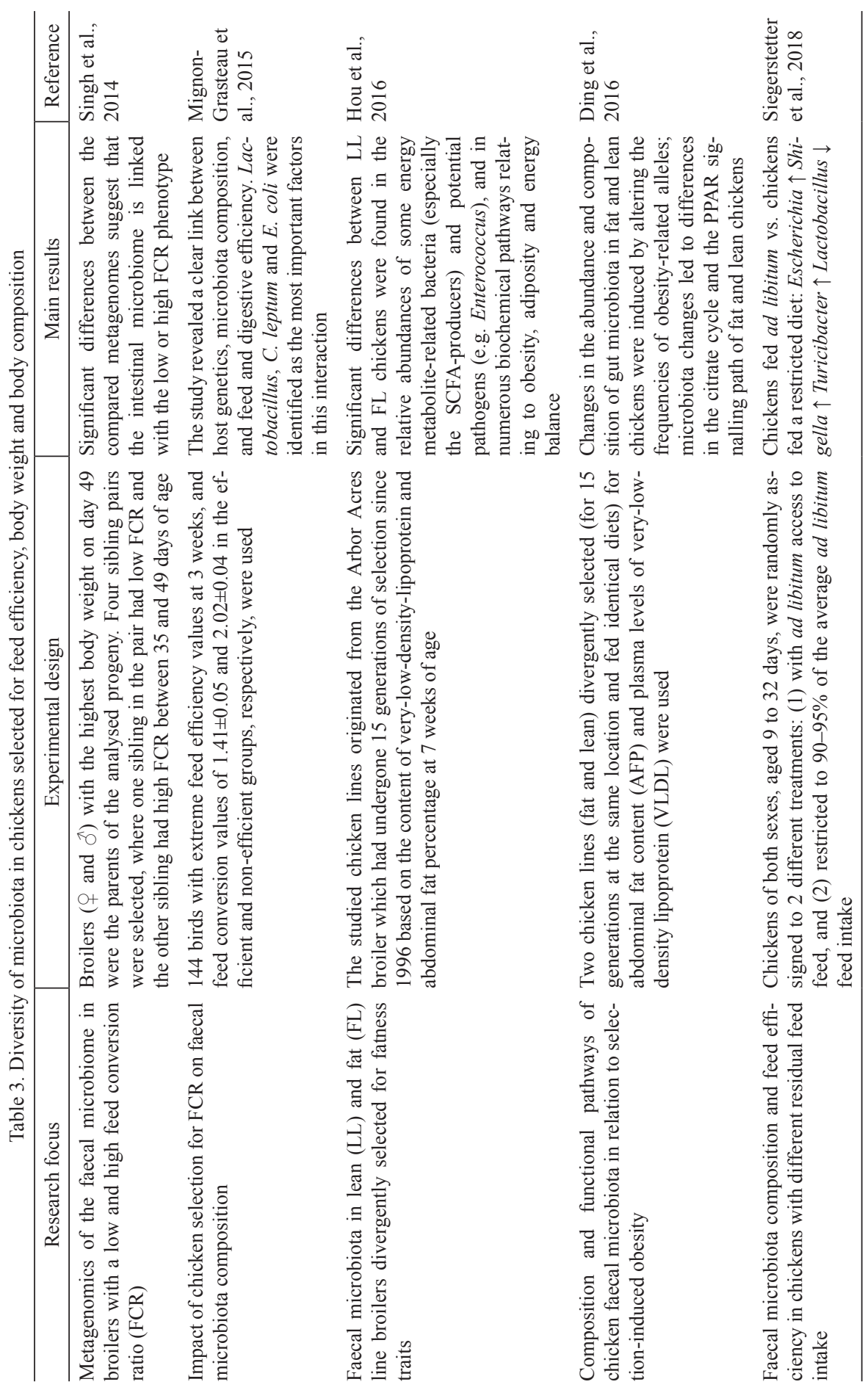




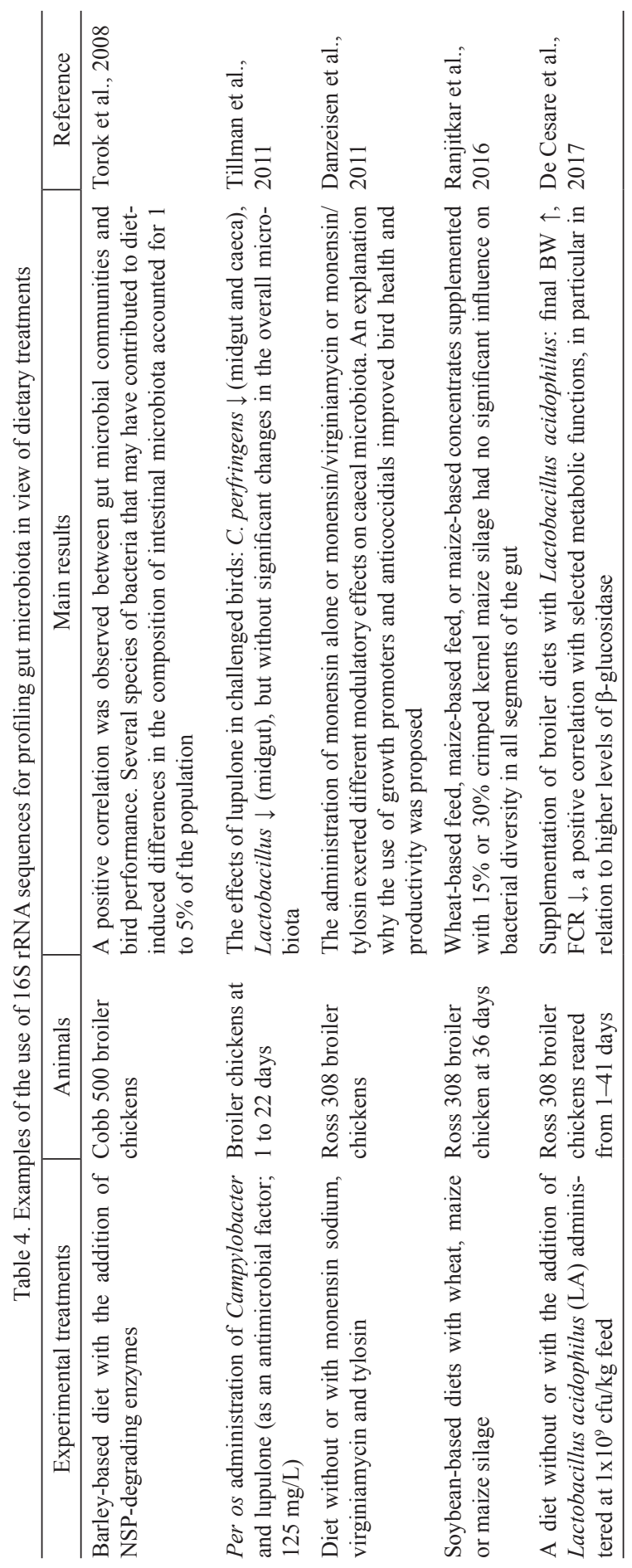


New analytical methods are more effective than traditional microbiological techniques in determining the variations in intestinal microbiota. Recent research indicates that digestive tract microbiota consists of more than 500 phylotypes or around 1 million bacterial genes, which is 40-50 times higher in comparison with the chicken genome (Wei et al., 2013; Sergeant et al., 2014; Oakley et al., 2014). According to estimates, the gastrointestinal microbiota in poultry is composed of 640 (Apajalahti et al., 2004) or even 900 (Wei et al., 2013) identified species, but only 10\% of these species have been cultured and characterised under laboratory conditions (Torok et al., 2011). The sequences identified in the gastrointestinal tracts of both poultry and humans represented 13 bacterial phyla (Wei et al., 2013) with a predominance of Firmicutes and Bacteroidetes, of which 10 accounted for a small part of the community (Oakley et al., 2014). Firmicutes are predominant in the upper gastrointestinal tract (approx. 90\% of the microbial community), and are represented mostly by Lactobacillus, Clostridium and Streptococcus (Wei et al., 2013). In the gut, the proportion of Firmicutes was estimated at $44-55 \%$, the proportion of Bacteroidetes, represented by the genus Bacteroides was estimated at $22-45 \%$, whereas Actinobacteria (including Bifidobacterium) and Proteobacteria (including Escherichia and Shigella) were far less abundant (Qu et al., 2008). Other authors give different values. In a study by De Cesare et al. (2017), the caecal digesta in chickens was also dominated by Firmicutes (93.9\%), whereas the proportions of Proteobacteria (1.74\%), Actinobacteria $(0.92 \%)$, Bacteroidetes $(0.35 \%)$ and Tenericutes $(1.18 \%)$ were considerably smaller. In the work of Borda-Molina et al. (2016), six bacterial families, including five members of the phylum Firmicutes and one member of the phylum Actinobacteria, accounted for $1 \%$ of the bacterial community in caecal samples.

According to Borda-Molina et al. (2018), the majority of metagenomics studies investigating the gastrointestinal tract of chickens have focused on caecal function, responses to pathogen challenge, the prominent role of microbiota in growth performance, comparisons of fat and lean lines, virulome and of antibiotic resistance genes. The experimental design and the results of the reviewed studies are presented in Tables 2-4. The studies cited in Table 2 revealed differences in the composition of ileal and caecal microbiota (Lu et al., 2003), demonstrated that microbial "maturation" takes place in the third week of a chicken's life (Ranjitkar et al., 2016), reported differences in the composition of microbiota in male and female broiler chickens (Lee et al., 2017), and demonstrated that environmental conditions and individual characteristics influenced the gut microbiota profile (Stanley et al., 2013). Recent studies have investigated intestinal microbiota in the context of biosecurity, housing conditions, litter, feed access and climate (Kers et al., 2018). Wei et al. (2018) compared the microbiomes of chickens and turkeys and reported differences in the species composition of their microbiota. The turkey microbiome was less diverse than the chicken microbiome, but in both bird species, Firmicutes, Bacteroidetes and Proteobacteria were the largest phyla that accounted for $>90 \%$ of all sequences. The predominant genera in both chicken and turkey sequences were Clostridium, Ruminococcus, Lactobacillus and Bacteroides, but their distribution differed in the analysed bird species. In turkeys, Campylobacter resided predominately in the caeca, and they were more abundant at 10 weeks of age and less abundant at slaughter (Wilkinson et al., 2017). 
The effect of intestinal microbiota on feed conversion in meat-type poultry is also a widely discussed problem in the literature. Poultry diets are composed mainly of cereals and high-protein components, and feed efficiency significantly affects the profitability of meat production. Feed accounts for $70 \%$ of total costs in the production of poultry meat (Willems et al., 2013), which is why the effect of intestinal microbiota on feed efficiency in poultry farming has been extensively studied. The results of experiments analysing the diversity of caecal microbiota in chickens with different feed efficiency, body weight and body composition are presented in Table 3. In a study by Singh et al. (2014), chickens with a high feed conversion ratio (FCR) were characterised by a higher abundance of the genera Acinetobacter, Bacteroides, Streptococcus, Clostridium and Lactobacillus, whereas birds with low FCR were colonised predominantly by Escherichia, Shigella and Salmonella. In an experiment by Mignon-Grasteau et al. (2015), the faecal digestibility coefficient of dry matter was genetically and positively correlated with L. crispatus, C. leptum and C. coccoides, and negatively correlated with $E$. coli. Lipid digestibility was negatively correlated with E. coli, and apparent metabolisable energy (AMEn) was positively correlated with $C$. coccoides and the $C$. coccoides to Lactobacillus ratio. Hou et al. (2016), Ding et al. (2016) and Siegerstetter et al. (2018) found that long-term selection for body weight, a lower content of abdominal fat, lower plasma levels of very-low-density lipoproteins and restricted feed intake influenced the composition of caecal microbiota. However, Hou et al. (2016) stressed that their results did not support the formulation of concrete conclusions regarding the influence of chicken gut microbiota on the metabolism of lean and fat line chickens. Further research is required to determine whether these bacteria are the cause or the consequence of the differences in feed utilisation (Pan and Yu, 2014). One of the consequences of the selection for feed intake in poultry can be an increased amount of readily fermentable components in the caeca (Walugembe et al., 2014), which can improve feed utilisation, increase intestinal weight and prolong caecal transit time (Rougière et al., 2002). For this reason, changes in the gut microbiota may result from feed intake and transit time in the gastrointestinal tract. Studies of humans demonstrated that population size of Firmicutes in faeces was positively correlated with the ability to recover energy from the diet (Jumpertz et al., 2011; Oakley et al., 2014) and that the Firmicutes to Bacteroides ratio could have important implications for health and nutrition. Studies of chickens revealed that a lower proportion of Firmicutes relative to Bacteroidetes in the caeca can promote effective utilisation of dietary energy. Sergeant et al. (2014) identified the sequences of more than 200 enzymes degrading non-starch polysaccharides in Bacteroidetes, which could be responsible for the utilisation of dietary energy in the lower gastrointestinal tract. Taxonomic analyses provide detailed information about the structure of microbial communities, but this knowledge is difficult to apply in evaluations of microbiota's influence on the host's body. A total of 59 bacterial genera were identified in the microbiome of chicken caeca, including 39 genera of the phylum Firmicutes, where the three most prevalent genera (Ruminococcus, Clostridium and Eubacterium) accounted for 5\% and the remaining genera represented up to $1 \%$ of the phylum Firmicutes (Wei et al., 2013). Bacteroidetes, the second most abundant phylum, was represented by 6 genera, including the 
predominant genus Bacteroides (approx. $40 \%$ of the population). The cited studies analysing the effect of microbiota on the utilisation of dietary energy in humans and chickens did not account for such considerable quantitative differences in bacterial genera, but compared only quantitative differences between the phyla Firmicutes and Bacteroides (Jumpertz et al., 2011; Bervoets et al., 2013; Oakley et al., 2014).

\section{Metagenomics from the physiological perspective}

Complex and cost-intensive metagenomics technologies enable the characterisation of microbial composition, but they fail to provide new information on the metabolites produced by gut microorganisms. Metagenomics procedures describe the accumulation of genomes and the corresponding genes in a given ecosystem, as well as the potential functionality of bacteria in specific environments (Marchesi and Ravel, 2015). According to estimates, around $20 \%$ of the genes in the metagenome of chicken intestinal microbiota control carbohydrate metabolism, $10 \%$ control protein and amino acid metabolism, and $1-2 \%$ are responsible for lipid metabolism (Qu et al., 2008). Microbial pathogens of the genera Salmonella, Clostridium, Campylobacter, Staphylococcus and E. coli pose a potential health risk for poultry (Oakley et al., 2014). Therefore, the mechanisms of competition between beneficial and pathogenic microflora, which involve competitive exclusion and the production of bacteriocins, are an important consideration (Yeoman et al., 2012). The composition of gut microbiota determines the functioning of the intestinal ecosystem (Table 5), and the composition and the physicochemical properties of the intestinal contents as well as the effects of microbial metabolites also play an important role (Zduńczyk et al., 2015). Polysaccharide fermentation processes play a crucial role in poultry caeca and in the colons of humans and monogastric animals, and they affect not only intestinal health, but the entire body (Stanley et al., 2014; Choi et al., 2015).

Table 5. Components of the intestinal ecosystem, factors affecting gut function, main metabolites and their physiological effects

\begin{tabular}{|c|c|}
\hline Ecosystem components & Impact factors \\
\hline Intestinal contents & $\begin{array}{l}\text { Diet: chemical composition and physicochemical properties (viscos- } \\
\text { ity, water content) } \\
\text { Enzymatic activity of the microbiota }\end{array}$ \\
\hline Intestinal microbiota & $\begin{array}{l}\text { Feed composition, mainly the content of non-starch polysaccharides } \\
\text { (NSP) } \\
\text { Bird genotype } \\
\text { Environmental conditions }\end{array}$ \\
\hline Metabolites & Effects \\
\hline $\begin{array}{l}\text { Short-chain fatty acids (SCFAs): } \\
\text { acetic, propionic, butyric and } \\
\text { other }\end{array}$ & $\begin{array}{l}\text { Local - intestinal integrity, inhibition of pathogen development, } \\
\text { absorption of } \mathrm{Ca}, \mathrm{Fe} \text { and } \mathrm{Mg} \text {, stimulation of the intestinal immune } \\
\text { system } \\
\text { Systemic - influence on lipid and glucose metabolism }\end{array}$ \\
\hline Lactic acid & Reduction in the $\mathrm{pH}$ of intestinal contents \\
\hline Bacteriocins & Bacteriostatic or bactericidal effects \\
\hline Peptides and neurotransmitters & $\begin{array}{l}\text { Stimulation of enteroendocrine cells (EECs), communication with } \\
\text { the central nervous system, influence on metabolism }\end{array}$ \\
\hline $\begin{array}{l}\text { Ammonia, amines, bile acid deg- } \\
\text { radation products and other }\end{array}$ & $\begin{array}{l}\text { Possible damage to intestinal mucosa, mucosal disorders, initiation } \\
\text { of neoplastic processes }\end{array}$ \\
\hline
\end{tabular}




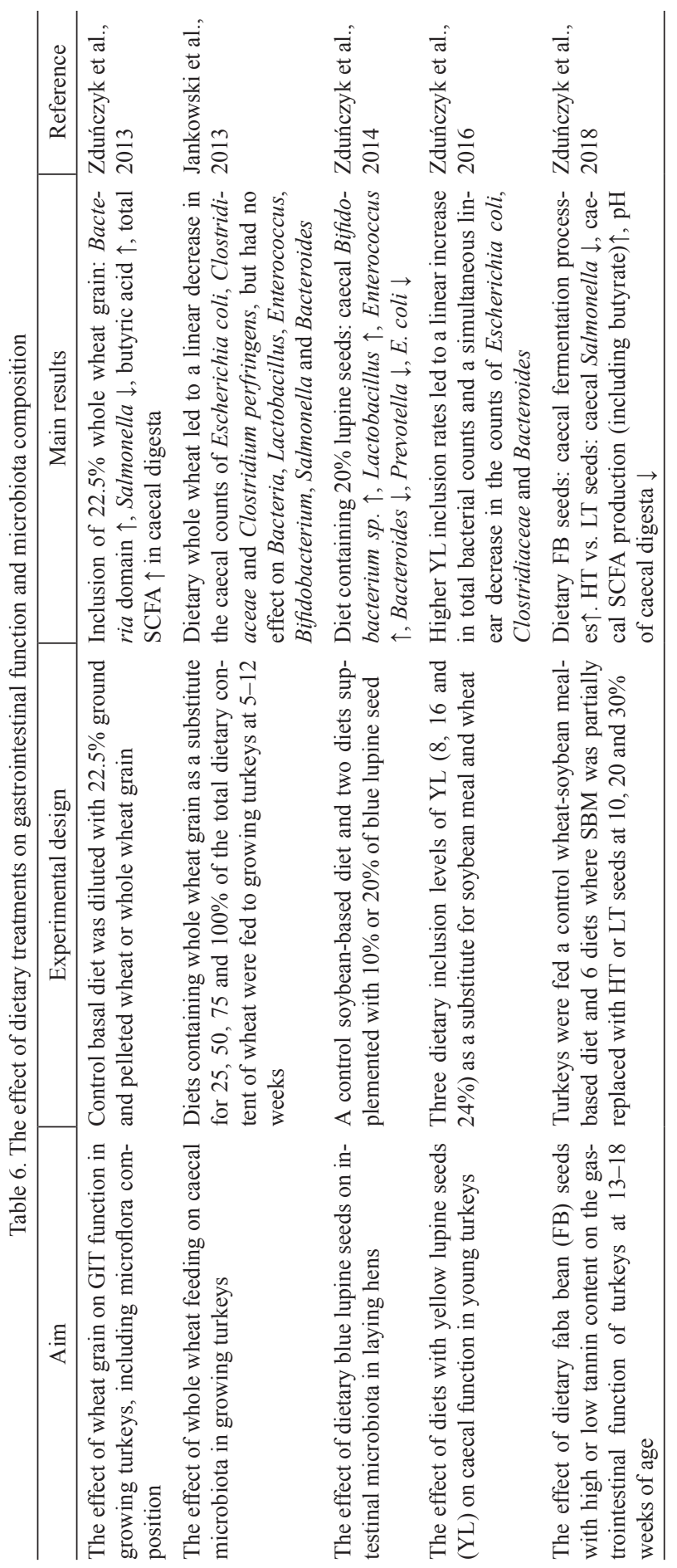


The metabolites produced by intestinal microbiota belong to three main groups of products: nutrients that are utilised by the host's body, including by the intestinal epithelium, to promote growth and enhance the function of short-chain fatty acids (SCFAs), including acetic acid, propionic acid and butyric acid; biologically active components (mainly B vitamins), and harmful and toxic substances which compromise nutritional benefits and pose a hazard to the host's health. These products exert local effects on competitive bacterial groups in the intestinal epithelium and the intestinal immune system (GALT - gut-associated lymphoid tissue) as well as systemic effects by influencing the host's metabolism (Table 5). Local effects include competitive inhibition of pathogenic microflora, protection of the intestinal mucosa and stimulation of the GALT response. In the group of SCFAs that exert systemic effects, acetic acid plays a key role by acting as a substrate in metabolic reactions, including the synthesis of cholesterol and long-chain fatty acids which regulate lipid metabolism (Millet et al., 2010). Butyric acid is a biomarker of intestinal health which delivers important local effects (De Maesschalck et al., 2015; Ducatelle et al., 2018).

The end products of protein fermentation by bacteria, including ammonia, phenols, indols, and amines, compromise gut health (Millet et al., 2010). Intestinal bacteria such as Clostridium, Enterococci and Bacteroides rely on proteins as an energy source, in particular when carbohydrates are not available in sufficient quantities in the intestinal contents. Therefore, high-protein diets can potentiate potentially pathogenic and pro-inflammatory microbiota (Yao et al., 2016). The mechanisms by which gut microbiota exert systemic and local effects, including competitive inhibition of pathogenic microflora, protection of intestinal mucosa, and GALT stimulation, have been described by other authors (Rehman et al., 2007; Den Besten et al., 2013; Pan and Yu, 2014; Stanley et al., 2014; Clavijo and Florez, 2018).

\section{Dietary methods for controlling the activity of intestinal microbiota}

In view of the potential benefits and risks of intestinal microbiota, numerous attempts have been made in the literature to control the development and activity of gut microorganisms by modifying poultry diets or feeding regimes (Zduńczyk et al., 2015). Various approaches and feed additives have been evaluated to inhibit or stimulate fermentation processes in the gastrointestinal tract of chickens, including a reduction in the dietary content of non-starch-polysaccharide (NSP)-degrading enzymes, addition of NSP-degrading enzymes, improvements in the physical structure of chicken diets, addition of readily fermentable oligosaccharides and polysaccharides (prebiotics), and the addition of viable bacterial cultures (available on the market as prebiotics). The effectiveness of these treatments has been assessed by numerous review articles in recent years (Huyghebaert et al., 2011; Hume, 2011; Oakley et al., 2014; Olnood et al., 2015; Ruiz et al., 2015; Clavijo and Florez, 2018). The results of few experiments where NGS methods were used to evaluate the effects of dietary modifications on poultry gut microbiota are briefly summarised in Table 6 . The composition of the chicken microbiome was less influenced by the incorporation of various cereals into poultry diets (Ranjitkar et al., 2016) and the addition of degrading enzymes (Torok et al., 2008) than by antibiotics (Danzeisen et al., 2011) and Campylobacter infection (Tillman et al., 2011). 
Metagenomics methods are expensive, which limits their availability for many researchers studying the gastrointestinal ecosystem (Borda-Molina et al., 2018). In the last decade, the effects of oligosaccharides, bacterial cultures and other factors on the composition of gut microbiota were investigated with the involvement of simpler techniques, including traditional culture methods, in many studies published in the literature, including in the renowned Poultry Science journal (Song et al., 2014; Zhao L. et al., 2013; Zhao P.Y. et al., 2016; Olnood et al., 2015). The results of own previous research into gut microbiota, including the major bacterial groups of Lactobacillus, Bifidobacterium, Escherichia coli and Clostridium, which relied on fluorescence in situ hybridization (FISH) and specific fluorescent probes for identifying DNA or RNA sequences, are presented in Table 5. In these studies, the activity of enzymes ( $\alpha$ - and $\beta$-glucosidase, $\alpha$ - and $\beta$-galactosidase, $\beta$-glucuronidase, $\alpha$-arabinopyranosidase, $\beta$-xylosidase and other) and the concentrations of SCFAs and ammonia, the key indicators of microbial enzymatic activity in the caeca, were also determined.

In a review article by Zduńczyk et al. (2016), partial replacement of ground wheat with wheat grain in the diets fed to turkeys induced desirable changes in their gut microbiota. In a study by Zduńczyk et al. (2013), the inclusion of $22.5 \%$ wheat grain in turkey diets improved gastrointestinal function, increased the percentage of Bacteria and Bifidobacterium spp., decreased the counts of Salmonella spp. in intestinal microflora, and increased the content of butyric acid and total SCFAs in the ceacal digesta. In an experiment conducted by Jankowski et al. (2013), gradual increase in the dietary content of wheat grain decreased the counts of Escherichia coli, Clostridiaceae and Clostridium perfringens. Favourable changes in the gut microbiota were also noted when soybean meal (SBM) was partially replaced with lupine or faba bean seeds, which decreased the content of raffinose family oligosaccharides and non-starch cereal polysaccharides. In laying hens, partial replacement of SBM with 20\% narrow-leaved lupine seeds increased the population size of Bifidobacterium and Clostridium spp., and decreased the counts of Escherichia coli and bacteria of the genera Bacteroides, Prevotella and Porphyromonas in the caecal contents (Zduńczyk et al., 2014). Desirable changes in the composition and enzymatic activity of gut microbiota led to an increase in the concentration of SCFAs and a decrease in ammonia levels and the $\mathrm{pH}$ value of caecal contents. In young turkeys fed diets with increasing levels of lupine seeds $(8,16$ and 24\%), a linear increase in the activity of bacterial glycolytic enzymes, an increase in the concentrations of SCFAs, and a decrease in the $\mathrm{pH}$ of caecal contents were observed relative to the group fed a soybean-based diet (Zduńczyk et al., 2016). A linear increase in total bacterial counts and a linear decrease in the populations of Escherichia coli, Clostridiaceae and Bacteroides were noted. Turkeys whose diets were supplemented with faba beans where characterised by higher counts of gut bacteria than turkeys fed soybeanwheat-based diets. Only the addition of low-tannin faba beans decreased the counts of Salmonella bacteria and increased the concentrations of volatile fatty acids, including butyric acid, in the intestinal contents of turkeys (Zduńczyk et al., 2018).

As demonstrated by the reviewed literature, new metagenomic techniques expand our understanding of the phylogenetic structure of gastrointestinal flora in 
poultry. In the cited studies, metagenomic methods supported the identification of previously unknown microorganisms that occur in small numbers. Next-generation sequencing provides indirect information about the quantitative structure of genes in gut microorganisms. There is a general scarcity of new information about microbial activity and the proportions of metabolites which affect intestinal integrity and the metabolism of the host organism. In this context, research studies are undertaken to obtain information about changes in the population size of the most important bacterial groups relative to the proportions of the key microbial metabolites in the intestinal contents of poultry, with the use of cheaper techniques. Further research is needed to characterise the composition of intestinal microbiota in greater detail based on complex, multi-faceted and dynamic relationships between the diet, the host organism and gut microbiota. The results will contribute to an improvement in feed efficiency and the welfare of birds in intensive poultry farming.

\section{References}

Apajalahti J., Kettunen A., Grahamh H. (2004). Characteristics of gastrointestinal microbial communities, with special reference to the chicken. World's Poultry Sci. J., 60: 223-232.

Barko P.C., Mc Michael M.A., Swans on K.S., Williams on D.A. (2018). The gastrointestinal microbiome: A Review. J. Vet. Intern. Med., 32: 9-25.

B ervoets L., Van Hoorenbeeck K., Kortleven I., Van Noten C., Hens N., Vael C., Goos sen s H., De s a ger K.N., Vankerckhoven V. (2013). Differences in gut microbiota composition between obese and lean children: a cross-sectional study. Gut Pathog., 5: 10.

Borda-Molina D., Vital M., S ommerfeld V., Rodehutscord M., Camarinha-Silva A. (2016). Insights into broilers' gut microbiota fed with phosphorus, calcium and phytase supplemented diets. Front. Microbiol., 7: 2033.

Borda-Molina D., Seifert J., Camarinha-Silva A. (2018). Current perspective of the chicken gastrointestinal tract and its microbiome. Comput. Struct. Biotechnol. J., 16: 131-139.

Campanaro S., Treu L., Kougias P.G., de Francisci D., Valle G., Angelidaki I. (2016). Metagenomic analysis and functional characterization of the biogas microbiome using high throughput shotgun sequencing and a novel binning strategy. Biotechnol. Biofuels, 9: 26.

$\mathrm{Ch}$ istoserdova L. (2009). Functional metagenomics: recent advances and future challenges. Biotechnol. Genet. Eng. Rev., 26: 335-352.

Cho I., B la se r M.J. (2012). The human microbiome: At the interface of health and disease. Nat. Rev. Genet., 13: 260-270.

Cho i K.Y., L e e T.K., S u 1 W.J. (2015). Metagenomic analysis of chicken gut microbiota for improving metabolism and health of chickens - a review. Asian-Australas. J. Anim. Sci., 28: 1217-1225.

Clavij o V., Flore z M.J.V. (2018). The gastrointestinal microbiome and its association with the control of pathogens in broiler chicken production: A review. Poultry Sci., 97: 1006-1021.

Cole J.R., Wang Q., Fish J.A., Chai B., McGarrell D.M., Sun Y., Brown T., PorrasAlfaro A., Kuske C.R., Tiedje J.M. (2014). Ribosomal Database Project: Data and tools for high throughput rRNA analysis. Nucleic Acids Res., 42: D633-D642.

Cruaud R., Vigneron A., Lucchetti-Miganeh C., Ciron P.E., Godfroy A., CambonB on avita M.A. (2014). Influence of DNA extraction method, 16S rRNA targeted hypervariable regions, and sample origin on microbial diversity detected by 454 pyrosequencing in marine chemosynthetic ecosystems. Appl. Environ. Microbiol., 80: 4626-4639.

Dan ze is en J.L., Kim H.B., I s a c s on R.E., Tu Z.J., J ohn s on T.J. (2011). Modulations of the chicken cecal microbiome and metagenome in response to anticoccidial and growth promoter treatment. PLoS One, 6: e27949.

De Cesare A., Sirri F., Manfreda G., Moniaci P., Giardini A.,Zampiga M., Meluzzi A. 
(2017). Effect of dietary supplementation with Lactobacillus acidophilus D2/CSL (CECT 4529) on caecum microbiome and productive performance in broiler chickens. PloS One, 12: e0176309.

De Maesschalck C., Eeckhaut V., Maertens L., De Lange L., Marchal L., Nezer C., De Baere S., Croubels S., Daube G., Dewulf J., Haesebrouck F., Ducatelle R., Taminau B., Van Immerseel F. (2015). Effects of xylo-oligosaccharides on broiler chicken performance and microbiota. J. Appl. Environ. Microbiol., 81: 5880-5888.

Den Besten G., van Eunen K., Groen A.K., Venema K., Reijngoud D-J., Bakker B.M. (2013). The role of short-chain fatty acids in the interplay between diet, gut microbiota, and host energy metabolism. J. Lipid Res., 54: 2325-234.

Deusch S., Tilocca B., Camarinha-Silva A., Seifert J. (2015). News in livestock research - Use of omics-technologies to study the microbiota in the gastrointestinal tract of farm animals. Comput. Struct. Biotechnol. J., 13: 55-63.

D ing J., Zha o L., Wang L., Zha o W., Zhai Z., Leng L., Wang Y., H e C., Zh a ng Y., Zhang H., L i H., M e n g H. (2016). Divergent selection-induced obesity alters the composition and functional pathways of chicken gut microbiota. Genet. Select. Evol., 48: 93.

Ducatelle R., Goossens E., De Meyer F., Eeckhaut V., Antonissen G., Haesebrouck F., Van Immers e e l F. (2018). Biomarkers for monitoring intestinal health in poultry: present status and future perspectives. Vet. Res., 849: 43.

Handelsman J., Rondon M.R., Brady S.F., Clardy J., Goodman R.M. (1998). Molecular biological access to the chemistry of unknown soil microbes: a new frontier for natural products. Chem. Biol., 5: R245-R249.

Hang J., D es a i V., Zavajevski N., Yang Y., Lin X., S a ty a R.V., Martinez L.J., Blaylock J.M., J a rm a n R.G., T ho m a s S.J., Ku s c hn e r R.A. (2014). 16S rRNA gene pyrosequencing of reference and clinical samples and investigation of the temperature stability of microbiome profiles. Microbiome, 2: 31.

H o o per L.V., Gord on J.I. (2001). Commensal host-bacterial relationships in the gut. Science, 292: 1115-1118.

Hou Q., Kwok L.Y., Zheng Y., Wang L., Guo Z., Zhang J., Huang W., Wang Y., Leng L., Li H., Zhang H. (2016). Differential fecal microbiota are retained in broiler chicken lines divergently selected for fatness traits. Sci. Rep., 6: 37376.

Hume M.E. (2011). Historic perspective: Probiotics, prebiotics, and other alternatives to antibiotics. Poultry Sci., 90: 2663-2669.

Huyghebaert G., Ducatelle R., van Immerseel F. (2011). An update on alternatives to antimicrobial growth promoters for broilers. Vet. J., 187: 182-188.

Jankowski J., Zduńczyk Z., Mikulski D., Przybylska-Gornowicz B., Sosnowska E., Juśki ew ic z J. (2013). Effect of whole wheat feeding on gastrointestinal tract development and performance of growing turkeys. Anim. Feed Sci. Technol., 185: 150-159.

Jumpertz R., Le D.S., Turnbaugh P.J., Trinidad C., Bogardus C., Gordon J.J., Krakoff J. (2011). Energy-balance studies reveal associations between gut microbes, caloric load, and nutrient absorption in humans. Am. J. Clin. Nutr., 94: 58-65.

Kers J.G., Velkers F.C., Fischer E.A., Hermes G.D., Stegman J.A., Smidt H. (2018). Host and environmental factors affecting the intestinal microbiota in chickens. Front. Microbiol., 9: 235 .

Kunin V., Engelbrektson A., Ochman H., Hugenholtz P. (2010). Wrinkles in the rare biosphere: Pyrosequencing errors can lead to artificial inflation of diversity estimates. Environ. Microbiol., 12: 118-123.

Lee K-C., Kil D.Y., Sul W.J. (2017). Cecal microbiome divergence of broiler chickens by sex and body weight. J. Microbiol., 55: 939-945.

Liu L., Li Y., Li S., Hu N., He Y., Pong R., Lin D., Lu L., Law M. (2012). Comparison of next-generation sequencing systems. BioMed Res. Int., 2012: 251364.

Lu J., I dris U., Harmon B., Ho facre C., Maurer J.I., L e e M.D. (2003). Diversity and succession of the intestinal bacterial community of the maturing broiler chicken. Appl. Environ. Microbiol., 69: 6816-6824.

Marchesi J.R., Ravel J. (2015). The vocabulary of microbiome research: a proposal. Microbiome, 3: 31 . 
Meyer A., Todt C., Mikkelsen N.T., Lieb B. (2010). Fast evolving 18S rRNA sequences from Solenogastres (Mollusca) resist standard PCR amplification and give new insights into mollusk substitution rate heterogeneity. BMC Evol. Biol., 10: 70.

Mignon-Grasteau S., Narcy A., Rideau N., Chantry-Darmon C., Boscher M-Y., Sellier N., Chabault M., Konsak-Ilievski B., Le Bihan-Duval E., Gabriel I. (2015). Impact of selection for digestive efficiency on microbiota composition in the chicken. PLOS One, 10: e0135488.

Millet S., van Oeckel M.J.,Aluwé M., Delezie E., De Brabander D.L.(2010). Prediction of in vivo short-chain fatty acid production in hindgut fermenting mammals: problems and pitfalls. Crit. Rev. Food Sci. Nutr., 50: 605-619.

Mohinudeen C., Joe M.M., B en son A., Tongmin S. (2017). An overview of Next-Generation Sequencing (NGS) technologies to study the molecular diversity of genome. Microbial Applications, $1: 295-317$.

Nielsen H. B., Almeida M., Juncker A.S., Rasmussen S., Li J., Sunagawa S., et al. (2014). Identification and assembly of genomes and genetic elements in complex metagenomic samples without using reference genomes. Nat. Biotechnol., 32: 822-828.

Oakley B.B., Lillehoj H.S., Kogut M.H., Kim W.K., Pedroso M.A., Lee M.D., Collett S.R., J o h n s on T.J., Cox N.A. (2014). The chicken gastrointestinal microbiome. FEMS Microbiol. Lett., 360: 100-112.

Olnood C.G., B eski S.S.M., Ij i P.A., Choct.M. (2015). Delivery routes for probiotics. Effects on broiler performance, intestinal morphology and gut microflora. Anim. Nutr., 1: 192-202.

$\mathrm{Pan}$ D., Yu Z. (2014). Intestinal microbiome of poultry and its interaction with host and diet. Gut Microbes, 5: 108-119.

Qu A., Brulc J.M., Wils on M.K., Law B.F., Theoret J.R., Joens L.A., Konkel M.E., Angly F., Dinsdale E.A., Edwards R.A., Nels on K.E., White B.A. (2008). Comparative metagenomics reveals host specific metavirulomes and horizontal gene transfer elements in the chicken cecum microbiome. PLoS One, 3: e2945.

Ranjitkar S., Lawley B., Tannock G., Engberg R.M. (2016). Bacterial succession in the broiler gastrointestinal tract. Appl. Environ. Microb., 82: 2399-2410.

Rehman H., Vahjen W., Awad W.A., Zentek J. (2007). Indigenous bacteria and bacterial metabolic products in the gastrointestinal tract of broiler chickens. Arch. Anim. Nutr., 6: 319-335.

Rougière N., Malbert C.H., Ridea u N., Cognié J., Carré B. (2002). Comparison of gizzard activity between chickens from genetic $\mathrm{D}+$ and $\mathrm{D}$ - lines selected for divergent digestion efficiency. Poultry Sci., 91: 460-467.

Ruiz R., Peinado M.J., Aranda-Olmedo I., A becia L., Suarez-Pereira E., Ortiz Melle t C., Garc i a F ern and e z J.M., R u bi o L.A. (2015). Effect of feed additives on ileal mucosaassociated microbiota composition of broiler chickens. J. Anim. Sci., 93: 3410-3420.

S alanitro J.P., Fairchild s I.G., Zgornicki Y.D. (1974). Isolation, culture characteristics, and identification of anaerobic bacteria from the chicken cecum. Appl. Microbiol., 27: 678-687.

S chlos s P.D., Handels m an J. (2003). Biotechnological prospects from metagenomics. Curr. Opin. Biotechnol., 14: 303-310.

Scholz M.B., Lo C.C., Chain P.S. (2012). Next generation sequencing and bioinformatic bottlenecks: the current state of metagenomic data analysis. Curr. Opin. Biotech., 23: 9-15.

S erge ant M.J., Constantinidou C., Cogan T.A., B ed ford M.R., P enn C.W., Pallen M.J. (2014). Extensive microbial and functional diversity within the chicken cecal microbiome. PloS One, 9: e91941.

Sharp to n T.J. (2014). An introduction to the analysis of shotgun metabolomic data. Front. Plant Sci., 209: $1-14$.

Siegerstetter S-C., Petri R.M., Magow an E., Lawlor P.G., Zebeli Q., O'Connel N.E., Metzler-Zebeli B.U. (2018). Feed restriction modulates the fecal microbiota composition, nutrient retention, and feed efficiency in chicken divergent in residual feed intake. Front. Mibrobiol. 9: 2698.

Singh K.M., Shah T.M., Reddy B., Deshpande S., Rank D.N., Joshi C.G. (2014). Taxonomic and gene-centric metagenomics of the fecal microbiome of low and high feed conversion ratio (FCR) broilers. J. Appl. Genet., 55: 145-154. 
Sitnicka D., Figurska K., Orzechowski S. (2010). Functional analysis of genes. Adv. Cell Biol., 2: 1-16.

Song J., Xi a o K., Ke Y.L., Ji a o L.F., Hu C.H., Dia o Q.Y., Shi B., Zou X.T. (2014). Effect of a probiotic mixture on intestinal microflora, morphology, and barrier integrity of broilers subjected to heat stress. Poultry Sci., 93: 581-588.

Stanley D., Geier M.S., Hughes R.J., Denman S.E., Moore R.J. (2013). Highly variable microbiota development in the chicken gastrointestinal tract. PLoS One, 8: e84290.

Stanley D., Hughes R.J., Moore R.J. (2014). Microbiota of the chickens gastrointestinal tract: Influence on health, productivity and disease. Appl. Microbiol. Biotechnol., 98: 4301-4310.

Thom as T., Gil bert J., M e y e r F. (2012). Metogenomics - A quide from sampling to data analysis. Microb. Inform. Exp., 2: 3.

Till m a n G.E., H a a s G.J., W is e M.G., O a kl e y B., S m ith M.A., S ir a g u s a G.R. (2011). Chicken intestine microbiota following the administration of lupulone, a hop-based antimicrobial. FEMS Microbiol. Ecol., 77: 395-403.

Torok V.A., Ophei K., Loo M., Hughes R.J. (2008). Application of methods for identifying broiler chicken gut bacterial species linked with increased energy metabolism. Appl. Environ. Microbiol., 74: 783-791.

Torok V.A., Hughes R.J., Mikkelsen L.L., Perez-Maldonado R., Balding K, Macalpine R., Percy N.J., O p he l-Keller K. (2011). Identification and characterization of potential performance-related gut microbiotas in broiler chickens across various feeding trials. Appl. Environ. Microbiol., 77: 5868-5878.

Tremaroli V., Bäckhed F. (2012). Functional interactions between the gut microbiota and host metabolism. Nature, 489: 242.

Turnbaugh P.J., Ley R.E., Hamady M., Fraser-Liggett C.M., Knight R. Gordon J.I. (2007). The human microbiome project. Nature, 449: 804-810.

Vincent A.T., Derome N., Boyle B., Culley A.I., Charette S.J. (2017). Next-generation sequencing (NGS) in the microbiological world: how to make the most of your money. J. Microbiol. Meth., 138: 60-71.

Walugembe M., Rothschild M.F., Persia M.E. (2014). Effects of high fiber ingredients on the performance, metabolizable energy and fiber digestibility of broiler and layer chicks. Anim. Feed Sci. Tech., 188: 46-52.

Wang W-L., X u S-Y., Ren Z-G., Ta o L., Ji ang J-W., Zheng S-S. (2015). Application of metagenomics in the human gut microbiome. World J. Gastroentero., 21: 803-814.

We i S., Morris o n M., Yu Z. (2013). Bacterial census of poultry intestinal microbiome. Poultry Sci., 92: 671-683.

Wilkin son T.J., Cow an A.A., Vall in H.E., O n i me L.A., O y a ma L.B., C a meron S.L., Gonot C., Moorby J.M., Waddams K., Theobald V.J., Leemans D., Bowra S., Nix ey C., Huws S.A. (2017). Characterization of the microbiome along the gastrointestinal tract of growing turkeys. Front Microbiol., 8: 1089; doi: 10.3389/fmicb.2017.01089. eCollection 2017.

Willems O.W., Miller S.P., Wood B.J. (2013). Aspects of selection for feed efficiency in meat producing poultry. World's Poultry Sci. J., 69: 77-88.

Ya o C.K., Mu ir J.G., Gi ib s on P.R. (2016). Review article: insights into colonic protein fermentation, its modulation and potential health implications. Aliment Pharmacol. Ther., 43: 181-196.

Yeoman C.J., Cjia N., Jeraldo P., Sipos M., Goldenfield N., White B.A. (2012). The microbiome of the chicken gastrointestinal tract. An. Health Res. Rev., 13: 89-99.

Zduńczyk Z., Jankowski J., Mikulski D., Przybylska-Gornowicz B., Sosnows ka E., Juśki ewicz J. (2013). Gastrointestinal morphology and function in turkeys fed diets diluted with whole grain wheat. Poultry Sci., 92: 1799-1811.

Zduńczyk Z., Jankowski J., Rutkowski A., Sosnowska E., Drażbo A., Juśkiewicz J. (2014). The composition and enzymatic activity of gut microbiota in laying hens fed diets supplemented with blue lupine seeds. Anim. Feed Sci. Technol., 191: 57-66.

Zduńczyk Z., Jankowski J., Ka czmarek S., Juśkiew i c z J. (2015). Determinants and effects of postileal fermentation in broilers and turkeys part 1: Gut microbiota composition and its modulation by feed additives. World's Poultry Sci. J., 71: 37-47. 
Zduńczyk Z., Krawczyk M., Mikulski D., Jankowski J., Przybylska-Gornowicz B., Juśkiewicz J. (2016). Beneficial effects of increasing dietary levels of yellow lupine (Lupinus luteus) seed meal on productivity parameters and gastrointestinal tract physiology in eight-week-old turkeys. Anim. Feed Sci. Technol., 211: 189-198.

Zduńczyk Z., Mikulski D., Jankowski J., Przybylska-Gornowicz B., Sosnowska E., Juśkiewicz J., Słomiński B.A. (2018). Effects of dietary inclusion of high- and lowtannin faba bean (Vicia faba L.) seeds on microbiota, histology and fermentation processes of the gastrointestinal tract in finisher turkeys. Anim. Feed Sci. Technol., 240: 184-196.

Zhao L., Wang G., S iegel P., He C., Wang H., Zhao W., Zhai Z., Tian F., A ha o J., Zhang H., Sun Z., Chen W., Zhang Y., Meng H. (2013). Quantitative genetic background of the host influences gut microbiomes in chickens. Sci. Rep., 3: 1-6.

Zha o P.Y., Li H.L., M oh a m madi M., Kim H. (2016). Effect of dietary lactulose supplementation on growth performance, nutrient digestibility, meat quality, relative organ weight, and excreta microflora in broilers. Poultry Sci., 95: 84-89.

Received: 8 VIII 2018

Accepted: 29 I 2019 